\title{
Gait Analysis from a Single Ear-Worn Sensor: Reliability and Clinical Evaluation for Orthopaedic Patients
}

\author{
Delaram Jarchi, Benny Lo, Charence Wong, Edmund Ieong, Dinesh Nathwani and \\ Guang-Zhong Yang, Fellow, IEEE,
}

\begin{abstract}
Objective assessment of detailed gait patterns after orthopaedic surgery is important for post-surgical followup and rehabilitation. The purpose of this paper is to assess the use of a single ear-worn sensor for clinical gait analysis. A reliability measure is devised for indicating the confidence level of the estimated gait events, allowing it to be used in free-walking environment and facilitating clinical assessment of orthopaedic patients after surgery. Patient groups prior to or following anterior cruciate ligament (ACL) reconstruction and knee replacement were recruited to assess the proposed method. The ability of the sensor for detailed longitudinal analysis is demonstrated with a group of patients after lower limb reconstruction by considering parameters such as temporal and force-related gait asymmetry derived from gait events. The results suggest that the ear-worn sensor can be used for objective gait assessments of orthopaedic patients without the requirement and expense of a formal gait analysis laboratory. It significantly simplifies the monitoring protocol and opens the possibilities for home-based remote patient-assessment.
\end{abstract}

Index Terms-gait, rehabilitation, Singular Spectrum Analysis (SSA), e-AR (ear-worn activity recognition) sensor.

\section{INTRODUCTION}

$\mathbf{G}$ Gait analysis as a tool in orthopaedic surgery ranges from simple observation of gait in a clinical setting to sophisticated tracking in dedicated gait laboratories. It has been used in both preoperative and postoperative settings, playing an increasingly important role in preoperative planning and objective assessment of functional outcomes [1] [2]. Quantitative gait analysis is now an integral part of common surgical workflows including joint arthroplasty, correction osteotomies and surgeries for children with Cerebral Palsy (CP) [3] [4]. It has been used for evaluating recovery of patients following major lower limb trauma [5] and reducing the risk of injuries [6]. Advances in sensing technologies have also enabled the development of miniaturised sensors, either wearable or positioned in the ambient environment, for providing real-time information that can be wirelessly interrogated [7]-[15]. One common approach to clinical gait analysis is to use multiple optical or inertial sensors affixed to the patient body for detailed bio-motion and gait analysis. The complexity of sensor placement and issues related to consistent sensor placement have limited these methods only to dedicated laboratory settings, requiring the support of a highly trained technical team. The possibility of using a single sensor for detailed gait assessment has many advantages, particularly in terms of the ease of clinical adoption, patient compliance, and the possibility of remote monitoring of patients in home environment. This has been used for quantitative gait pattern analysis [16]-[19] and assessment of

D. Jarchi and B. Lo are with the Hamlyn Center, Imperial College London, London, UK (e-mail: d.jarchi@imperial.ac.uk, benny.lo@imperial.ac.uk). E. Ieong and D. Nathwani are with Imperial College Healthcare NHS Trust, London, United Kingdom, (e-mail: edieongortho@gmail.com, d.nathwani@imperial.ac.uk). G-Z. Yang is with the Hamlyn Centre, Imperial College, London, UK (g.z.yang@imperial.ac.uk). patients with Parkinson's disease (PD) [20], [21]. Thus far, a range of studies have been carried out by using different wearable sensors for gait analysis. These are listed in Table I ( [5], [8], [12]-[14], [16], [20]-[25]). For example, the ear-worn Activity Recognition (eAR) sensor has been used in both experimental and clinical settings [5], [12], [22]-[25]. The sensor includes an accelerometer and the major advantage of the sensor is in its bio-inspired design and ease of consistence sensor placement. Although some other wearable sensors such as Inertial Measurement Units (IMUs) can provide more information such as an easier way of computing more gait parameters (e.g. spatial gait parameters), here, we investigate the possibility of using a single accelerometer based sensor for large scale patient populations and inside a clinical environment while looking into clinically relevant parameters. This will enable construction of a reliable system in the future for continuous home-based monitoring in which an accelerometer preferred over the use of gyroscopes due to their unavoidable drift.

For pathological gait analysis, one major question is the accuracy of the system outputs when there are large patient-specific gait variations. In previous analysis schemes, gait patterns are normally assessed in a controlled environment while patients walked on an instrumented treadmill. This also simplifies the analysis framework as all movements are well controlled and repetitive. With the current drive in assessing free-walking gait patterns to better reflect a patient's quality of movement and subject specific recovery process, the analysis algorithms need to differentiate step-by-step variations and adapt to subject-specific changes. The purpose of this paper is to propose an adaptive method that is generalisable to different patient groups and can improve the overall reliability of the gait analysis system. This is practically important especially for patient populations walking in a free walking environment where the recorded signals may not show proper periodicity and clear distinction of right and left gait events is difficult. As an example, acceleration signals from two patients with knee replacement walking on a treadmill and a corridor are shown in Fig. 1. In situations such as gait assessments outside the gait laboratory, inherent reliability evaluation of the system outputs is essential to the practical use of the system in clinical settings.

\section{Materials And Methods}

\section{A. Hardware Setup}

The e-AR sensor developed by Sensixa is used for this study. It contains a 3D accelerometer (Analog Devices ADXL335), an 8051 processor with a 2.4-GHz transceiver (Nordic nRF24E1), a 2-MB EEPROM (Atmel AT45DB161), and a 55-mAhr LiPolymer [26]. The sensor is ergonomically designed and proven to be robust in capturing gait related acceleration signals from either the left or right ear [26], [27]. The sensor signal can be stored or wirelessly interrogated on a tablet. In this study, a dedicated software environment (BSN Analytics) running on Windows 8 has been used. It allows realtime visualisation and processing of detailed gait signals, as well as capturing individual patient information that can be interfaced to electronic medical records. The platform also permits synchronised video recording that can be used as a reference or ground truth for subsequent data analysis. The system setup of the hardware platform is shown in Fig. 2. The designed algorithm for data analysis in this research has been implemented in MATLAB (The Mathworks Inc.) 
for off-line data processing. However, the designed algorithm is under development to be integrated into the Tablet as an App for real-time and online data processing.

For analysis of force-related gait asymmetry, a pair of pressure insoles (PAROTEC, Paromed, Germany) has been used to provide reference data for validating the e-AR sensor and associated analysis algorithm. The PAROTEC system requires a controller that must be placed around the waist [28] and each insole has a height of about $3 \mathrm{~mm}$ and contains 24 microsensors mounted under a constrained hydrocell. Both insoles are connected to the controller using two cables. The PAROTEC system is used in this study to observe left/right gait asymmetry from the e-AR sensor versus the ground reaction forces (GRFs) given by the insoles.

\section{B. Algorithm Design}

The accuracies of gait events estimated from the e-AR sensor by the proposed algorithms [12] [22] are assessed using an instrumented treadmill [12] and pressure insoles for walking in a corridor [22]. For initial detection of gait events, the method based on singular spectrum analysis (SSA) and longest common subsequent algorithm (LCSS) [12] with its extension [22] has been used. This gait event detection algorithm is mainly based on the SSA algorithm in which the acceleration signals from different axes are converted into matrix forms by using delayed versions of the input accelerations. After applying singular value decomposition (SVD) to the resulted matrices, selected output subspaces from appropriate axes are used for various purposes such as trend removal and extraction of dominant oscillation. Then using all information from all axes, and also applying the LCSS algorithm, left and right temporal gait events are obtained [12], [22]. The algorithm for estimating gait events is described in the following steps:

1) Set the embedding dimension as $l$,

$\mathbf{s}=\left[s_{0}, \ldots, s_{n-1}\right] \longleftarrow$ acceleration signal,

2) Convert $\mathbf{s}$ into trajectory matrix $\mathbf{X}=\left[\begin{array}{cccc}s_{0} & s_{1} & \ldots & s_{k-1} \\ s_{1} & s_{2} & \ldots & s_{k} \\ \vdots & \vdots & \ddots & \vdots \\ s_{l-1} & s_{l} & \ldots & s_{n-1}\end{array}\right]$

where $k=n-l+1$.

3) Apply SVD onto the $\mathbf{X}$ to find elementary matrices $\mathbf{X}_{i}\left(\sqrt{\lambda_{i}}, \mathbf{u}_{i}, \mathbf{v}_{i}\right)$,

4) Select the indices of $i$ and group elementary matrices $\sum_{i} \mathbf{X}_{i}$ for trend removal and extraction of dominant oscillation (from sum of anterior-posterior (AP) and super-inferior (SI) axes),

5) Apply LCSS, SVD and peak detection techniques using all axes and the information from the previous steps to estimate left and right

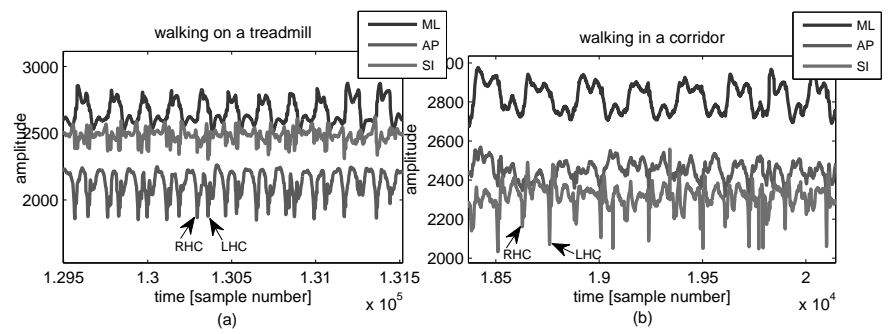

Fig. 1. (a) A patient with knee replacement walking on a treadmill. (b) Another patient with knee replacement walking in a corridor. For walking on a treadmill more distinctive signal patterns from the AP axis are usually obtained because the walking condition is well controlled. By examination of the right and left heel contacts (RHCs and LHCs), for a pathological patient temporal and amplitude asymmetry of the gait signals can be observed from the AP and SI axes which affect the periodicity of the gait signals. A useful axis which can provide supplementary information in the estimation of gait events is the ML axis and used in this study for re-analysis and post-processing of the estimated gait events for their evaluation outside gait laboratories. temporal gait events.

The major gait events considered in Jarchi et al. [12], [22] include heel contact and toe-off events where toe-off events are estimated after detection of heel contacts by applying LCSS algorithm. A better precision in detecting heel contacts leads to more accurate results for estimation of toe-off events. Here, a reliability measure is introduced, which is based on evaluating the correctness of estimated right and left heel contacts. It is obtained by post-processing of acceleration data and estimated heel contacts to find the cases where there are over-estimated or under-estimated heel contact events.

1) Detection of over-estimated gait events: The Medio-lateral (ML) axis corresponds to left-right accelerations and has been used for left/right discrimination of gait events which are estimated mainly based on the peaks from the SI and AP axes [29]. Based on this, using the characteristics of the ML axis and the prior knowledge, it is possible to re-evaluate the correctness of the estimated right and left gait events and find situations where there are over-estimated/underestimated gait events.

For detecting over-estimated gait events, the analysis is, then, performed using the ML axis of the e-AR sensor. By segmenting the accelerations from the ML axis, similar gait patterns can be observed from right heel contacts (RHCs) to the left heel contacts (LHC). In addition, similar gait patterns can be obtained by considering the ML accelerations from LHCs to the RHCs. In Fig. 3, a sequence of gait events is shown. The effect of asymmetrical walking patterns that occur with most orthopaedic patients before or after surgery can be detected from the difference in signal amplitudes of the AP and SI axes during the RHCs and LHCs. This also affects the periodicity of gait patterns. As long as asymmetrical walking is not causing postural instability, the ML axis can produce the same patterns for cycles segmented from right-to-left heel contacts and also same patterns separately for cycles segmented from left-to-right heel contacts. By considering one full cycle of the gait using the ML axis from one RHC to the next one (see Fig. 3), it is possible to detect whether there are extra estimated heel contacts in between right heel contacts that lead to different segmentation results on the ML axis. The idea for detection of extra estimated heel contacts is to construct a phase space from right-to-left and also left-to-right heel contact segmentations of the ML axis. By simultaneously under-sampling and matching of each two consecutive segmented cycles from right-to-left heel contacts, we can form a matrix of two vectors. One vector is the under-sampled

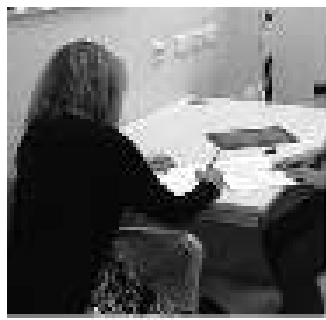

$1+$

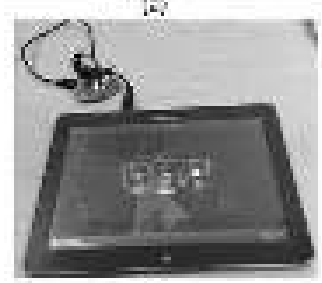

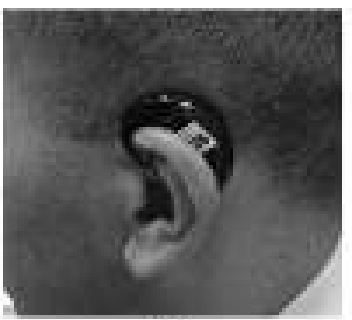

;b)

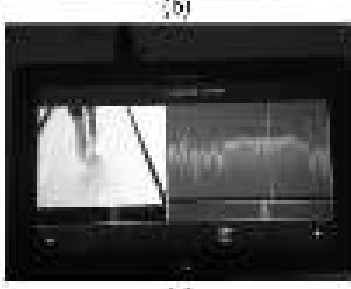

Fig. 2. (a) A patient inside the clinic signing the consent form to participate in the e-AR experiment. (b) The e-AR sensor worn by a male patient. (c) The sensor and the tablet used for data collection. (d) Synchronised e-AR accelerations and video frames given by the tablet's camera for a patient walking in the corridor. The interface of the implemented software to record the e-AR accelerations integrated with video frames called BSNAnalytics is shown. 
cycle of the ML axis from the first right-to-left heel contact. The other vector is negative of the corresponding under-sampled cycle of the $\mathrm{ML}$ axis for the next right-to-left contact (see $\mathbf{M}_{i}$ in Algorithm 1). Inclusion of the negative sign is helpful to analyse two types of segmentations (right-to-left and left-to-right) which will be shown in the following. To perform under-sampling and point matching, a delay of $\tau$ and $\widetilde{\tau}$ are assigned by considering the maximum length of two consecutive gait cycles from right-to-left heel contacts. Based on this, each two consecutive right-to-left heel contacts form the $\mathbf{M}_{i}$ matrix. All the matrices considering right-to-left segmentations are combined to form the M matrix. Then, Eigen-decomposition is applied to the covariance matrix of $\mathbf{M}$. After representing $\mathbf{M}$ as a $2 \times 2$ matrix with two vectors constructing each row, its covariance will then be a $2 \times 2$ matrix that each of its diagonal elements is equal to 1 due to separate normalisation of each row of the $\mathbf{M}$ matrix. The sum of eigenvalues will be equal to 2 (i.e. the trace of the covariance of $\mathbf{M}$ ). In the case of perfect segmentation, the eigenvector with the larger eigenvalue must be $\frac{1}{\sqrt{(2)}}\left[\begin{array}{c}1 \\ -1\end{array}\right]$ where its eigenvalue $\lambda$ is equal to 2 that make the other eigenvalue to be equal to 0 . However, considering the gait cycles, in reality reaching an eigenvalue of 2 is practically not possible. To obtain an accurate segmentation, the resulted eigenvector with larger eigenvalue must be in the direction of $\frac{1}{\sqrt{(2)}}\left[\begin{array}{c}1 \\ -1\end{array}\right]$ and its eigenvalue should have a reasonably high value of close to 2. In a similar manner, the ML axis is segmented from all leftto-right heel contacts (see $\mathbf{N}_{i}$ and $\mathbf{N}$ matrices in Algorithm 1) and the corresponding Eigen-decomposition is performed. A threshold can be used to assign a low/medium/high/very-high level of confidence based on the direction of resulted eigenvectors and their eigenvalues as explained above (see $\lambda_{1}, v, \widetilde{\lambda}_{1}, \widetilde{v}$ parameters in the algorithm in Algorithm 1). The complete procedure for detection of over-estimated heel contacts and measuring a confidence level is shown in the algorithm in Algorithm 1. In this algorithm, $\mathbf{r}_{h c}$ and $\mathbf{l}_{h c}$ represent indices for right and left heel contacts respectively. The parameters $n$ and $m$ control under-sampling of the gait cycles. For this application we set these parameters to one. By increasing $n$ and $m$, fewer samples are obtained for constructing the phase space. Regardless of the obtained values for the eigenvalues if any of the resulted eigenvector is not in the expected direction $(\widetilde{v}<1$ or $v<1)$, the reliability will be set to low. Then, in the case of having ( $\widetilde{v}=1$ and $v=1$ ),

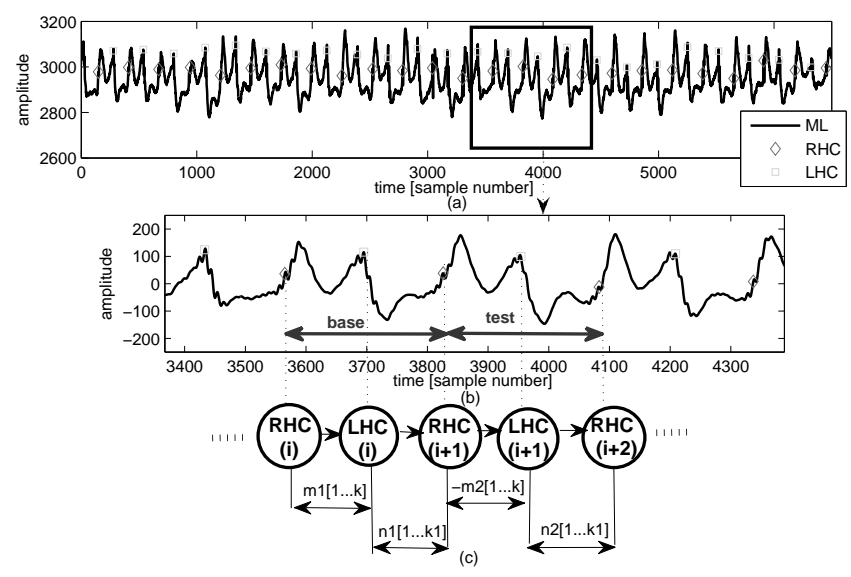

Fig. 3. (a) Raw accelerations and detected RHCs and LHCs on the ML axis. (b) One segment of the data from (a) after trend removal and detection of RHCs and LHCs. The blue arrows with labels of base and test show the parts of the signal (right-to-right heel contacts) used for detection of underestimated gait events (see Algorithm 2). (c) A sequence of gait events matched to the ML axis in (b). This plot shows how the vectors in $\mathbf{M}_{i}$ and $\mathbf{N}_{i}$ matrices are created to detect over-estimated gait events as summarized in Algorithm 1 .

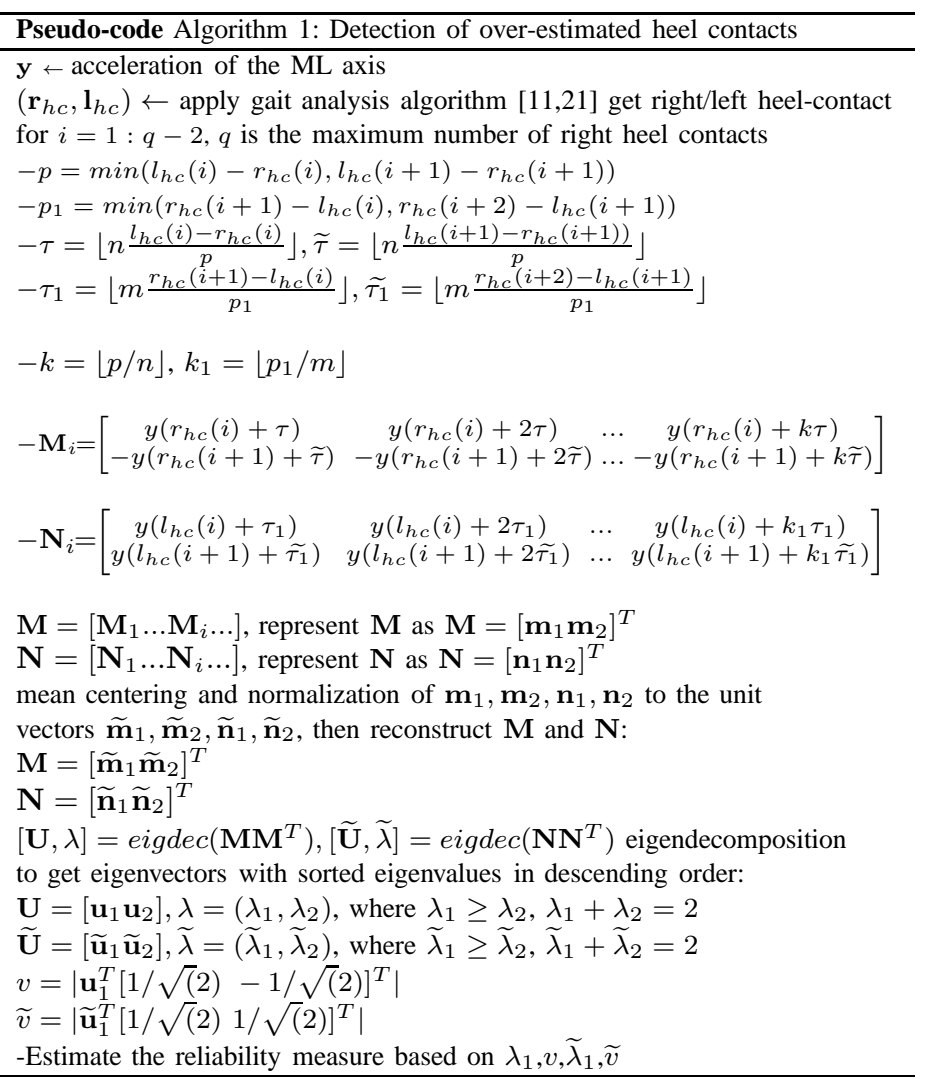

a threshold on each of the resulted eigenvalue will determine the estimated reliability. The bigger the eigenvalues will lead to a higher accuracy. In practice, a low reliability value is mostly related to the extra peaks detected using the dominant oscillation from the sum of $\mathrm{AP}$ and SI axes [12], [22]. Therefore, it is possible to discard most or all of extra peaks by using simple thresholding. All peaks detected on dominant oscillation of AP [12] and sum of AP and SI axes [22] with negative values (local minima with negative values) are selected as potential heel contacts. Wrongly detected heel contacts, which often occur for pathological gaits, are local minima with negative values that are close to zero. For removing those points, we set a threshold to be the average of all local minima.

2) Detection of under-estimated gait events: Detection of under-estimated gait events is difficult due to variations in gait speed and cadence across subjects. For detection of under-estimated gait events, the gait cycles from the ML axis are first interpolated to a maximum length considering all the gait cycles. Then each gait cycle from one RHC to the next is separated and its trajectory matrix is created to form a base trajectory matrix. This trajectory matrix is constructed using the embedding dimension and by delayed versions of the input segmented cycle of ML axis [30], [31]. The SVD is applied to the trajectory matrix of the base and the first $\mathrm{k}$ columns of the $\mathrm{U}$ matrix are selected to form the subspace of the base. The next consecutive cycle from right heel contacts is used to create the trajectory matrix of the test. The trajectory matrix of the test is projected to the subspace of the base. The distance of columns of the trajectory matrix of the test to the subspace spanned by the base is calculated by the relevant projection (see Algorithm 2 ). The under-estimated gait events are detected in places where the estimated distance is bigger than a threshold. The basic idea behind the proposed algorithm is based on a general change-point detection algorithm [32], [33] using SSA algorithm. The proposed algorithm for detection of segments related to under-estimated gait events is provided in Algorithm 2. The objective is to detect variations in right-to-right heel contacts and detect fundamental changes from one right-to-right heel contact to the next one. In the case of detecting such fundamental change, a segment of the data related to the under- 


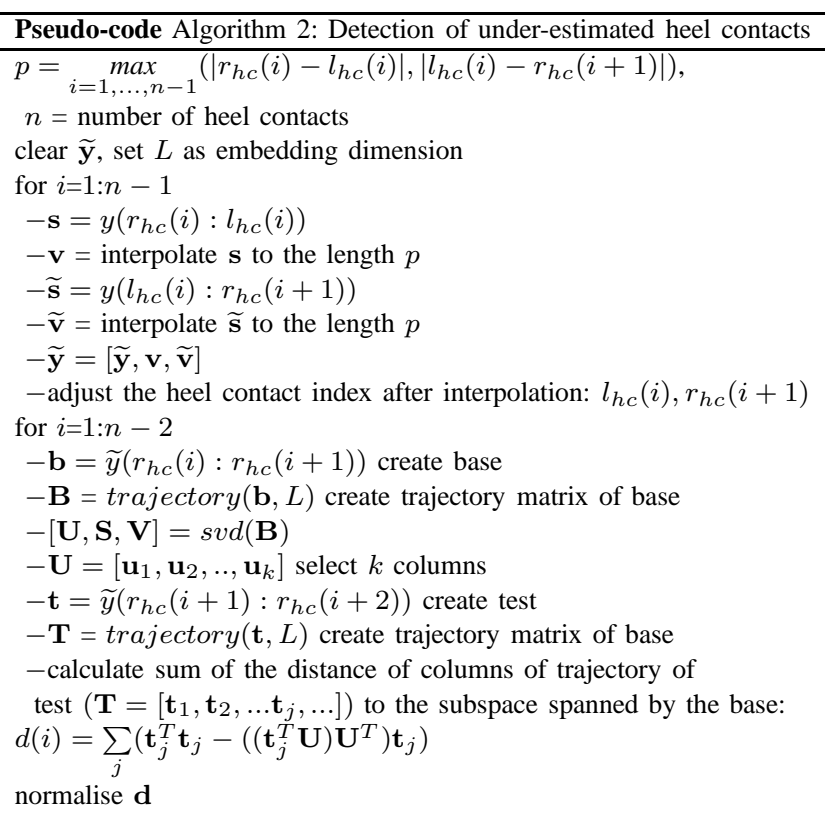

normalise $\mathbf{d}$

-Detect under-estimated heel contacts that $d$ value exceeds than a threshold

estimated gait events can be found. Then the estimation of gait parameters will be performed separately for the segments which do not contain under-estimated gait events. One interesting application of the change-point detection algorithm using SSA will be identification of freezing of the gait in PD patients which can be explored in future studies.

\section{RESULTS}

\section{A. Validation of gait asymmetry using PAROTEC}

For monitoring Orthopaedic patients, the gait asymmetry in terms of both timing and the amount of force is of great importance. Postoperative orthopaedic patients usually put more force into the uninjured foot. However, due to other factors, some patients (especially elderly patients and patients with bilateral knee replacement) may exert more force into the injured foot. Therefore, discrimination of the amount of left or right forces exerted is an important measurement. If the right and left heel contacts are estimated correctly, it is possible to find out the larger gait asymmetry towards left/right foot. To demonstrate the ability of the proposed algorithm for gait asymmetry detection using the e-AR sensor, a healthy subject equipped with $\mathrm{e}-\mathrm{AR}$ and PAROTEC systems was asked to walk in a corridor and exert a larger force towards the right foot. Another healthy subject was asked to walk and put more weight onto the left foot. Manual synchronization of the e-AR and PAROTEC systems has been performed by two big heel contacts at the beginning and at the end of the experiment. Synchronized recordings of the pressure insoles and e-AR accelerations were analysed to differentiate the left and right gait asymmetry level of the subjects. The results are shown in Fig. 4 where both accelerations, left and right GRFs given by the pressure sensors inside the left and right shoes are presented. The results for the first subject who exerted larger force towards the right foot are shown in Fig. 4 (a-c). It can be seen from e-AR accelerations that there is a larger peak at RHCs. Also, the sum of GRFs for the six sensors at the heel side of the insoles is shown in Fig. 4 (c) which shows larger forces for the right foot insole. The results for the second subject are shown in Fig. 4(d-f). In Fig. 4(e) the gait asymmetry as the larger amplitudes for the LHCs can be seen from SI and AP axes where the asymmetry seems to be more distinctive from the AP axis. Therefore, the gait asymmetry may not have effects only on SI axis. In addition, the gait asymmetry was visible for the second subject by summation of all the pressure sensors as shown in Fig. 4(f). For each gait cycle, we calculated the e-AR gait asymmetry level as the ratio of the acceleration magnitude at detected RHC to the LHC. While
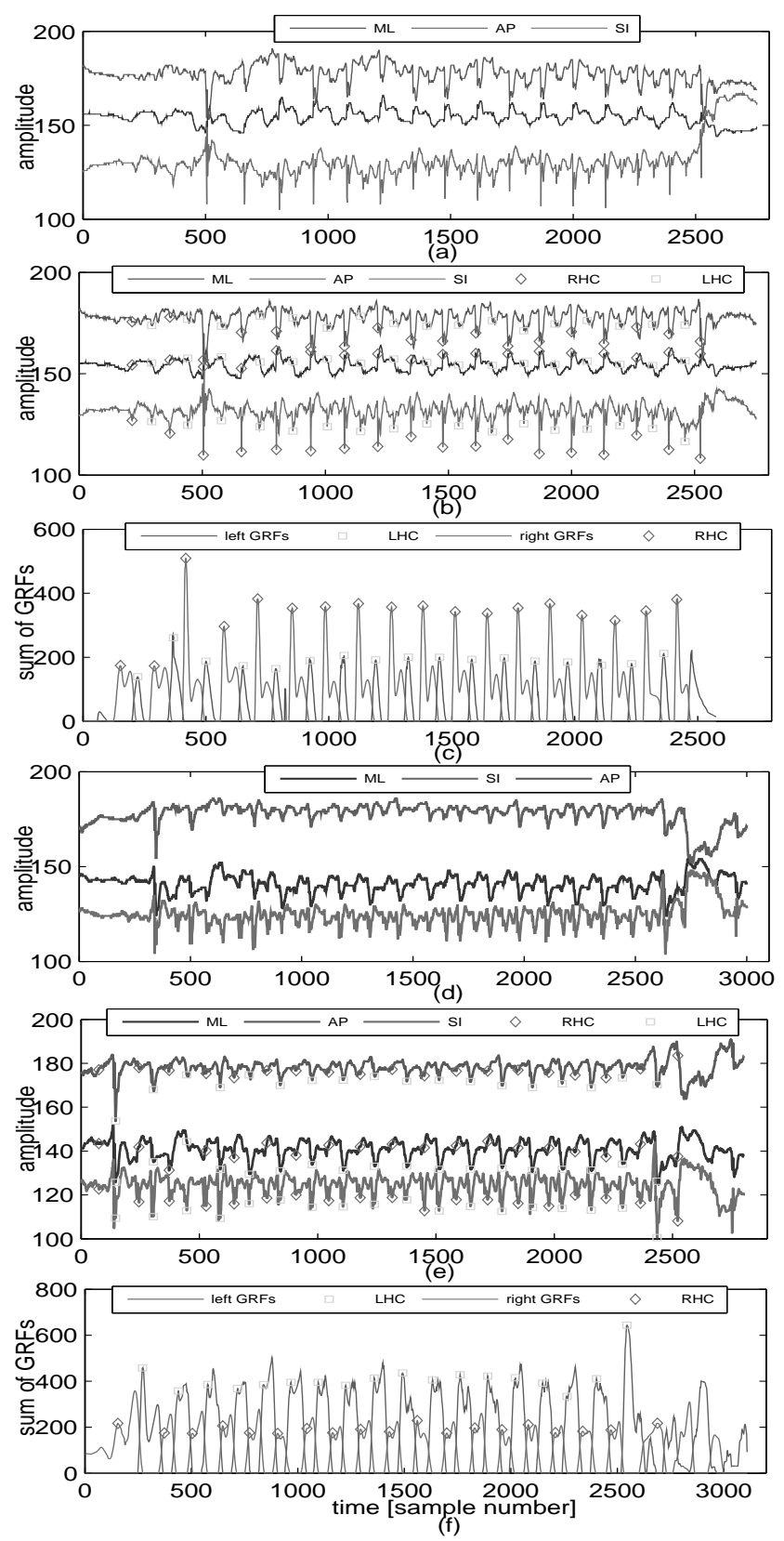

Fig. 4. (a) Raw e-AR accelerations for healthy subject 1 with larger force towards right foot. (b) Trend removed accelerations and detected RHCs and LHCs. (c) Sum of GRFs from six sensors at the heel side of the right and left insoles. (d) Raw e-AR accelerations for healthy subject 2 with larger force towards left foot. (e) Trend removed accelerations and detected RHCs and LHCs. (f) Sum of GRFs from 24 sensors of the right and left insoles.

for the PAROTEC system, it is the ratio of sum of right GRFs to the left GRFs following the touch of the foot by the ground. The results of gait asymmetry level for each gait cycle are shown in Fig. 5. In the top and bottom rows, the asymmetry is evaluated based on the SI axis and a combination of the SI and AP axes respectively. The mean square errors between estimated asymmetry from e-AR and PAROTEC systems were calculated and less error is observed by considering the asymmetry using the sum of the AP and SI axes. For the first subject, the mean square errors are 0.2766 for the SI axis and 0.1727 for the sum of AP and SI axes. For the second subject, the mean square errors are 0.1042 for the SI axis and 0.0837 for the sum of AP and SI axes. The estimations seem to be slightly better for using both SI and AP axes. 

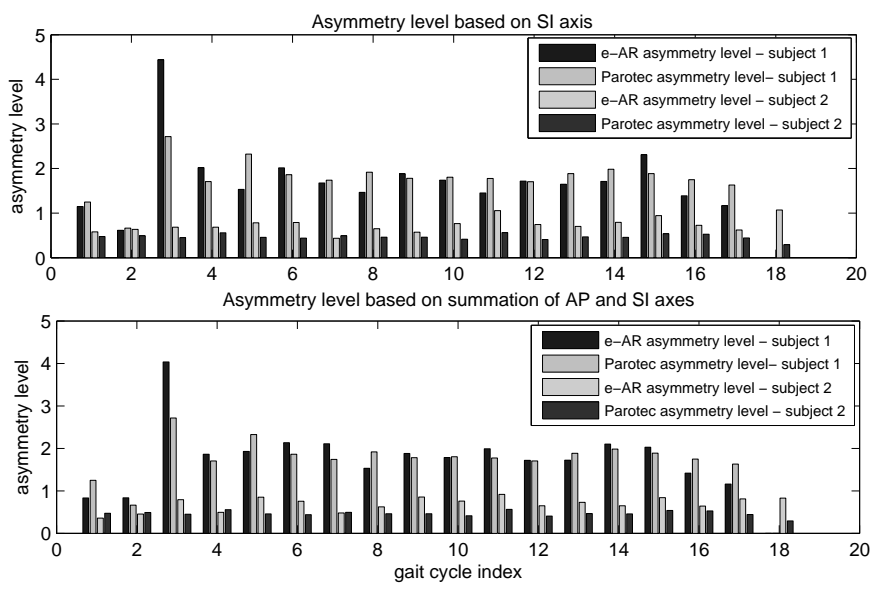

Fig. 5. Top row: Estimated asymmetry level using SI axis for healthy subjects 1 and 2. Bottom row: Estimated asymmetry level using both SI and AP axes for healthy subjects 1 and 2 .
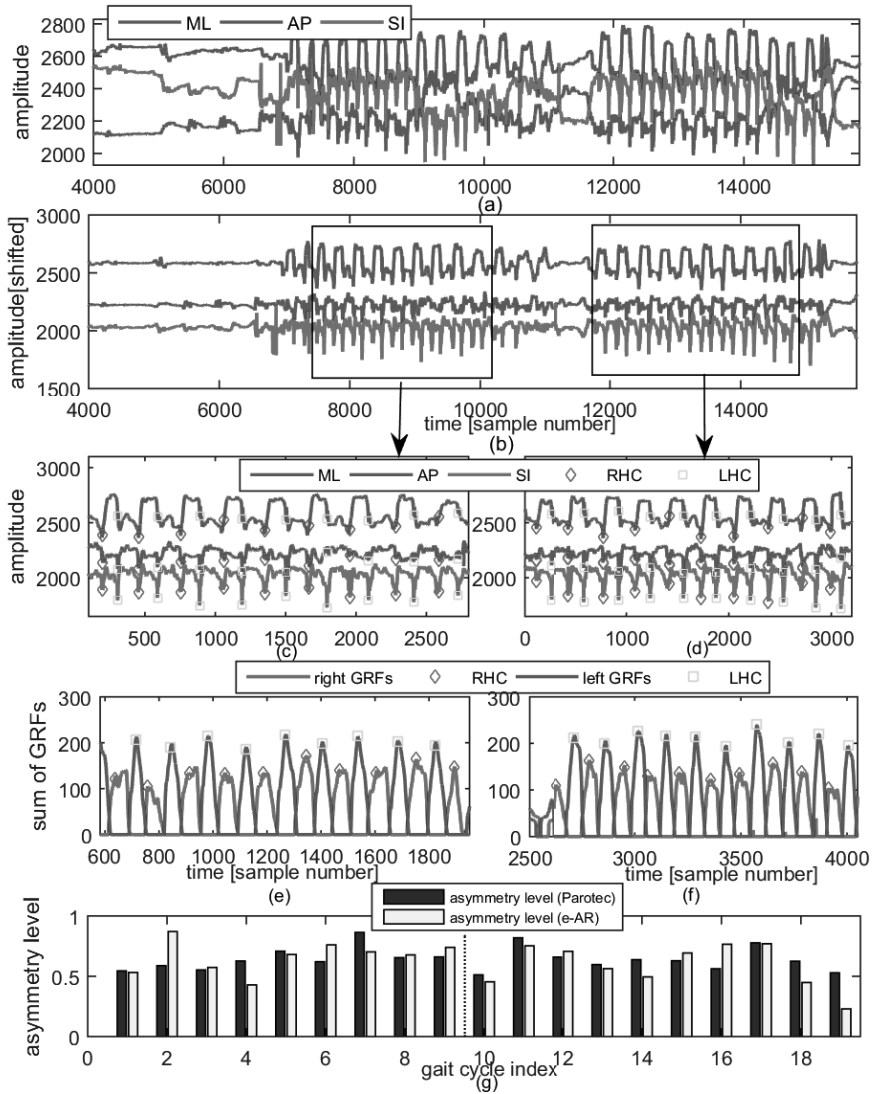

Fig. 6. (a) Recorded raw acceleration for a patient prior to right knee surgery. (b) Trend removed accelerations. (c) Detected RHCs and LHCs for the first trial. (d) Detected RHCs and LHCs for the second trial. (e) Sum of GRFs from six sensor at the heel side of the left and right sensors for the first trial. (f) Sum of GRFs from six sensor at the heel side of the left and right sensors for the second trial. (g) The estimated asymmetry level from e-AR and Parotec systems for each gait cycle. The first 9 cycles are related to the first trial and the second 10 cycles are related to the second trial.
To evaluate the accuracy of the estimated asymmetry level from eAR sensor using Parotec system as the reference platform, 8 patients were recruited in the study. The patients were equipped with both e$\mathrm{AR}$ and Parotec systems and asked to walk along $10 \mathrm{~m}$ long corridor of the fracture clinic at Charing Cross Hospital. Each patient performed the experiment in 2 trials. The results of the estimated asymmetry level (from sum of SI and SP axes) in each gait cycle for a selected patient prior to his surgery for right knee are shown in Fig. 6. The results of estimation for all eight patients are plotted in Fig. 7. In Fig. 7(a) the Bland-Altman plot is shown for the estimation from e-AR and Parotec systems. The mean difference of estimations is obtained as -0.0195 and standard deviation as 0.3192 . The mean absolute error is obtained as 0.2407 and the standard deviation as 0.2093 . The asymmetry level should be averaged for each patient to give an overall symmetry index. In Fig. $7(\mathrm{~b}, \mathrm{c})$ The average of the asymmetry level from e-AR and Parotec systems for each patient has been compared and shown. Based on the results, the e-AR sensor has been able to accurately discriminate the larger force for the left and right foot for all the patient in which the mean squared error of 0.0446 has been obtained for all the subjects. By increasing the number of trials we expect less error in the overall estimation of asymmetry level using the e-AR sensor.
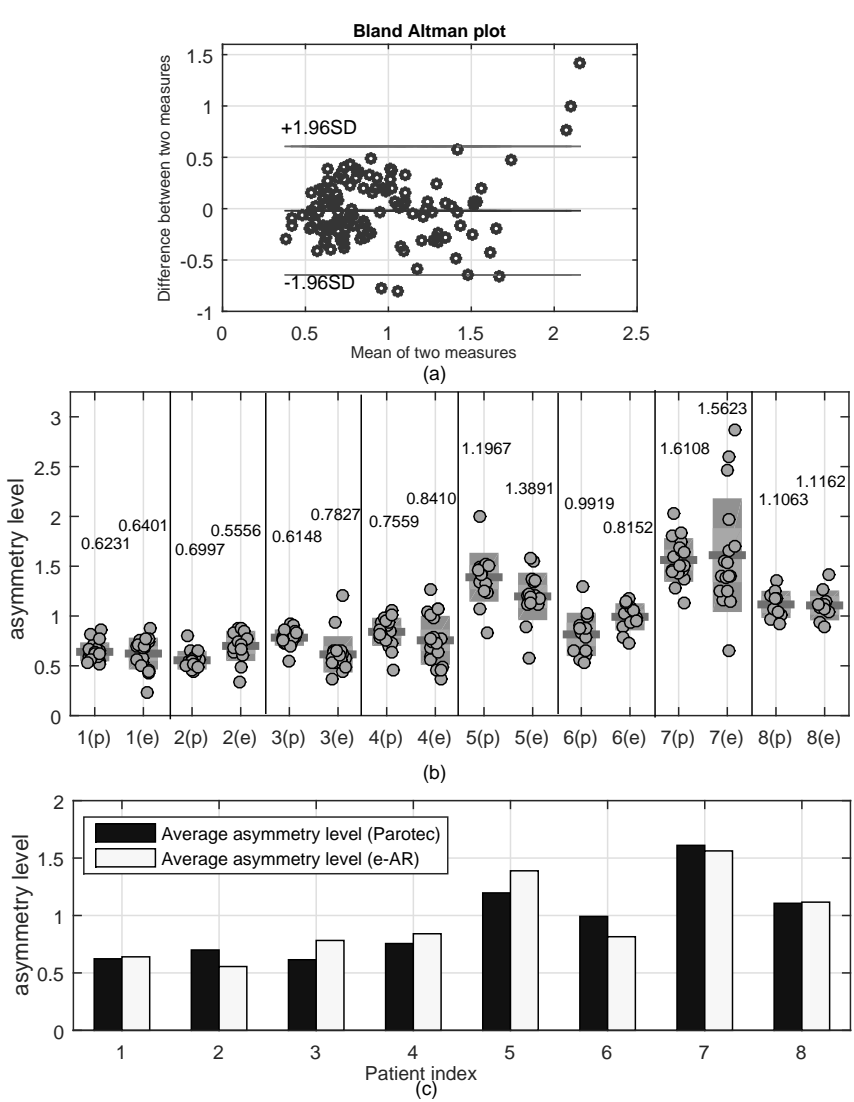

Fig. 7. (a) Bland-Altman plot for the estimated asymmetry level at each gait cycle from 8 orthopaedic patients using e-AR and Parotec systems. (b) For each patient the estimated asymmetry levels for all gait cycles using eAR sensor versus Parotec system are shown. The horizontal axis is related to the index of each patient which is followed by (e) and (p) representing the estimation from e-AR and Parotec systems respectively. The average asymmetry level for all gait cycles is calculated for e-AR and Parotec systems separately (redlines) and are shown in numbers for each patient. (c) The average estimated asymmetry level for the patients from the (b) plot. 

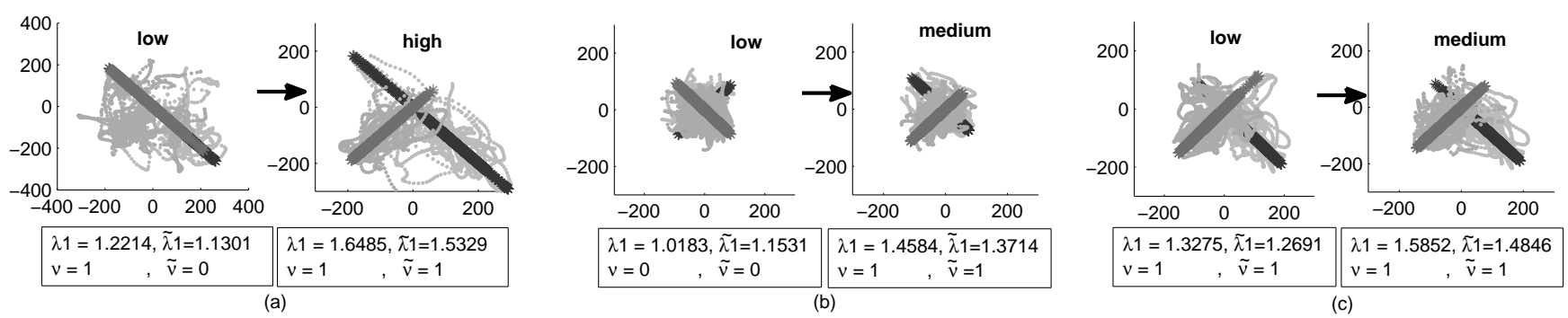

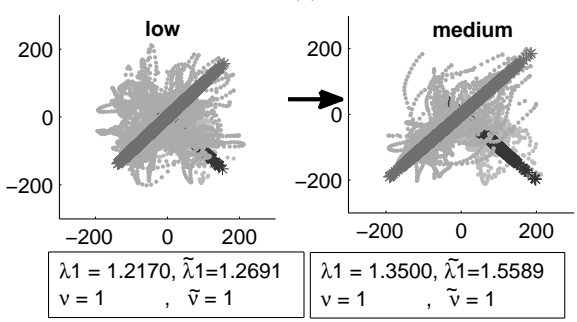

(d)

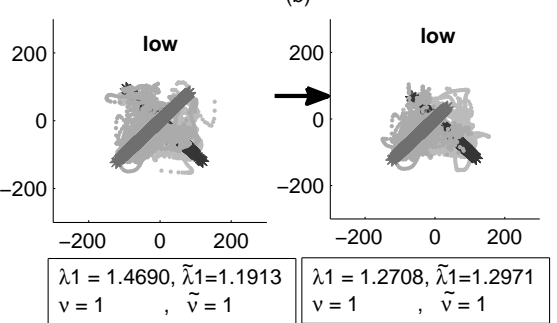

(e)

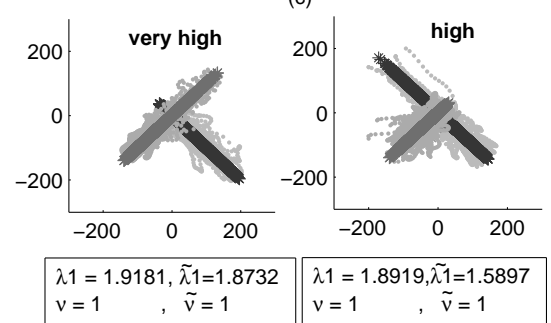

(g)

Fig. 8. Analysis of gait patterns from ML axis in a phase space. Blue and red colors are related to the right-to-left segmentations (M matrix) and left-to-right segmentations ( $\mathbf{N}$ matrix) respectively. The straight lines (darker blue and red lines) demonstrate the projected patterns on the direction of resulted eigenvectors (with bigger eigenvalue for each decomposition i.e. right-to-left and left-to-right). For each plot the values of the parameters $\lambda_{1}, v, \widetilde{\lambda}_{1}, \widetilde{v}$ in Algorithm 1 are shown. A value of the zero for $\widetilde{v}$ or $v$ demonstrate the wrong partitioning of the phase space for left/right segmentations (see (a) and (b)). (a) A reliability of low is converted to high. (b)-(d) A reliability of low is converted to medium. (e) The reliability of low has not been changed for new estimated gait events. This means the segment of the data should be changed or the experiment must be repeated. (f) A reliability of very high which shows large amount of the variances along the desired directions. (f) A reliability of high has been obtained.

\section{B. Assessment on patients with Anterior Cruciate Ligament $(A C L)$ reconstruction or knee replacement}

Data from 23 ACL patients and 31 patients with unilateral, bilateral and total knee replacement was collected for analysis. These patients were assessed inside the fracture clinic of the Charing Cross Hospital, London, UK. Based on the protocol, the patients while wearing only the e-AR sensor were asked to walk along two corridors of the clinic with a total of $40 \mathrm{~m}$ length at their selected speed. We have used mixture of patients having knee surgery either for ACL reconstruction and knee replacement to enrich and enhance our dataset containing acceleration signals for monitoring their recovery and gait performance following the surgery.

1) Detection of over-estimated gait events: For 50 patients, a window of fixed size of 10000 samples was selected for the gait analysis algorithm. For the remaining 4 patients smaller window size was selected in which the minimum segment size was 3000 samples due to a shorter walking time by these patients. The values of the parameters $\lambda_{1}, v, \widetilde{\lambda}_{1}, \widetilde{v}$ as the results of applying the algorithm in Algorithm 1, are shown for 7 selected patients in each plot of Fig. 8. For 4 patients (Fig. 8 (a, b, c and d)), a reliability of low is converted to medium/high after setting the threshold for peak detection from dominant oscillation to discard extra peaks. For one patient (Fig. 8(e)) the reliability of low is unchanged after trying to remove extra peaks from dominant oscillation. A reliability of high and very high is depicted in Fig. 8(f, g). It can be seen from these plots that $\widetilde{\lambda}_{1}$ and $\lambda_{1}$ are relatively high and $\lambda_{1}$ is very close to 2 for Fig. 8(f)). For Fig. 8(a), the eigenvectors for both decompositions are in the same directions, this means misplacement of a number of LHCs with RHCs or having extra RHCs between actual RHCs. As another example, in Fig. 8(b), the directions of the resulted eigenvectors demonstrate the misplacement of RHCs and LHCs.

2) Detection of under-estimated gait events: Examples for detecting segments of the data related to missing gait events or underestimated gait events using the proposed algorithm in Algorithm 2 are shown in Figs. (9-10). By analysing the described dataset, the threshold value on the vector $d$ in algorithm in Algorithm 2 was empirically set to 0.38. In Fig. 9(a) and Fig. 9(d), two and three segments of the data related to missing gait events are detected by analysing the ML axis, respectively. In Fig. 10(a-d), one segment of the missing gait events is visible while the AP axis shows noisy accelerations. After detecting under-estimated gait events, the gait parameters will be estimated based on the parts of the accelerations which do not contain missing gait events. In Fig. 11, the reliability estimates for a total of 54 ACL and knee replacement patients are shown. Based on the values of $\lambda_{1}$ and $\widetilde{\lambda}_{1}$, a rough partitioning of the reliability estimates are performed. Based on this, the results of the estimations for 35 patients are found to be high. Among these patients, one individual with a reliability of high had initially a reliability of low (see Fig. 8(a)). For 14 patients, the reliability of medium is obtained and 5 of them had previously a reliability of low in estimations ( 3 patients' gait patterns shown in phase space Fig. 8(bd)). Finally for 5 subjects, a reliability of low has remained unchanged for the selected segment. By cross-examination of the synchronised videos for all the patients with reliability of high or very-high, there are accurate estimations for the right and left heel contacts with no apparent over-estimation or under-estimation problem. In Table II, the reliability measure with medium and low values are evaluated in terms of final assessment of reliability measurements. It can be seen from Table II that for all patients with a reliability of low converted to medium, there are no over-estimated gait events by individual analysis of synchronised images and detected gait events on the accelerations; however, there are under-estimated gait events that were correctly detected by the algorithm in Algorithm 2. For subjects with reliability of low there are underestimation problems detected accurately only for patient \#19 (see Table II). For all the patients with medium reliability, there is no over-estimation problem. However, for four of them, the under-estimation problem remains. In addition, for all four patients with a borderline reliability of medium close to high (see Fig. 11), there is no over-estimation or underestimation problem. As shown in Table II, 17 out of 25 cases with under-estimation or over-estimation problem are correctly detected by the algorithm. Although quantification of detailed accuracies of gait events is not of interest in this study, overall, $68 \%$ improvement is obtained for correctly locating the left and right heel contacts from patients' populations for gait assessments in a clinical environment. For 5 subjects with reliability of low, two of them (\#19 and \#2) were walking with a stick (both patients were pre-operative). The other 

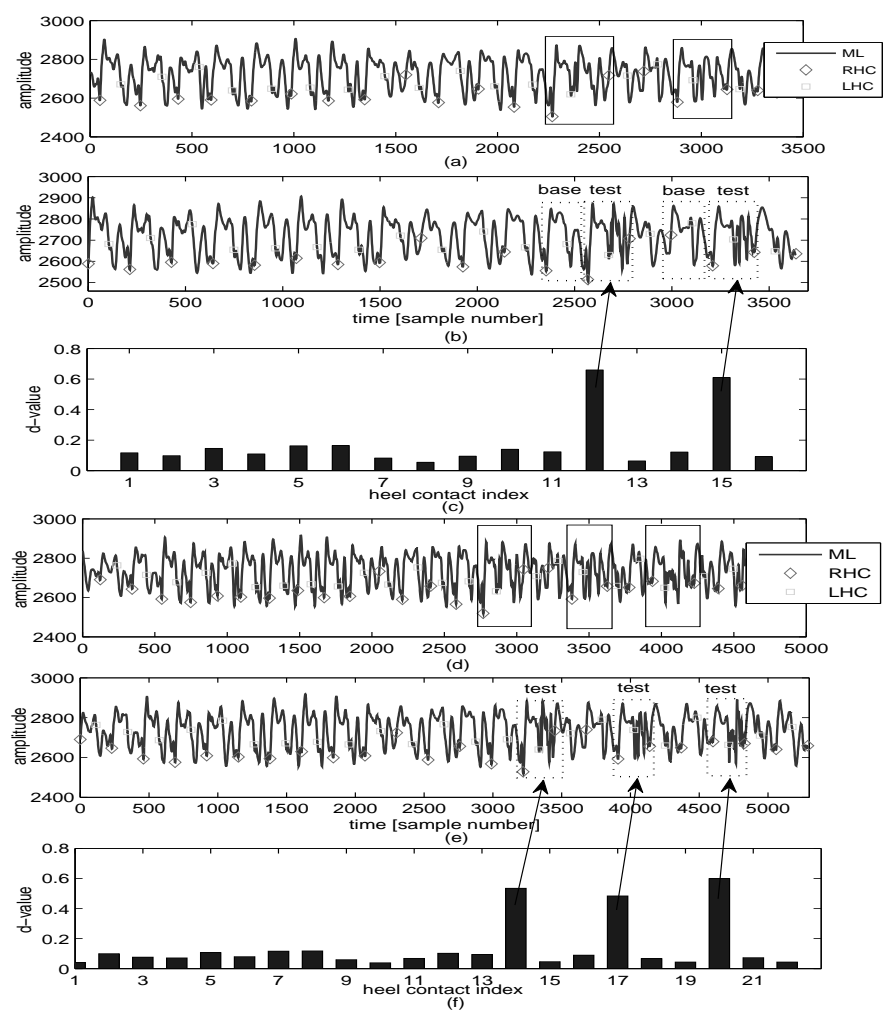

Fig. 9. Two examples for detection of under-estimated gait events. The ML accelerations and the estimated gait events plus the segments relating to missing gait events are shown in (a) and (d). The interpolated accelerations are shown in (b) and (e). The values of the vector of $\mathbf{d}$ in Algorithm 2 are shown in (c) and (f). The values in $\mathbf{d}$ vector which exceeds than 0.38 for are used to select segments of missing gait events.

three patients are:

- \#14 an elderly walking with a low speed (having short steps) with a bilateral knee replacement 2 years post surgery.

- \#34 an elderly 6-weeks post-operative.

- \#42 a patient pre-operative of knee replacement surgery.

It is very likely that walking with a stick and taking short steps (common among elderly population) increases the chance of getting a reliability of low. More improvements on the analysis algorithms can be performed in future studies.

\section{Monitoring patients with lower limb trauma}

To assess the generalizability of the algorithm for longitudinal analysis of patients over a long period of the recovery process, data from 16 patients following lower limb reconstruction was used. These data was acquired for one of our previous studies already published in [5] for the purpose of psychometric evaluation of a sensorbased mobility score. Based on the protocol the patients performed a 6-Minute walk along the corridor, Timed Up \& Go and Timed Up \& down stairs. In this study we have used the data regarding walking along the corridor. Eight patients performed the experiment in the clinic for all sessions exactly at 3, 6, 9 and 12 months postoperatively. The other eight patients attended one session or more, but not all required sessions. Three gait parameters including step time asymmetry (ratio of the right-to-left step time), amplitude asymmetry (ratio of the magnitude of acceleration using sum of AP and SI axes at RHC to the corresponding magnitude at LHC) and right stride time (timing from one RHC to the next one) were selected to evaluate monitoring of these patients following surgery. Since the perfect symmetry is reflected as one, the asymmetry values in Table III are expressed as the difference between one and the calculated asymmetry ratio. The values of these gait parameters for all subjects

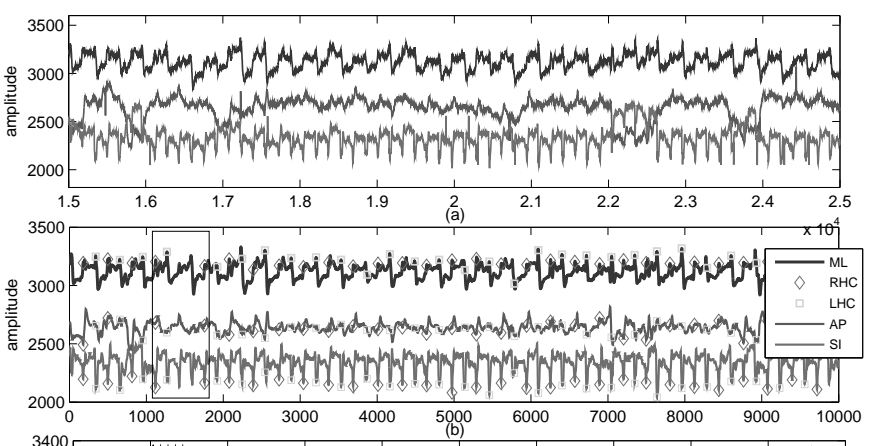

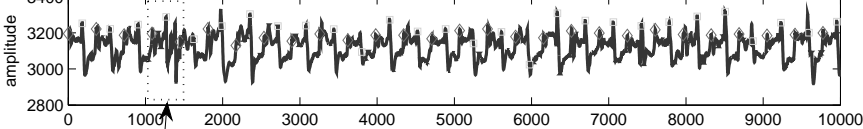

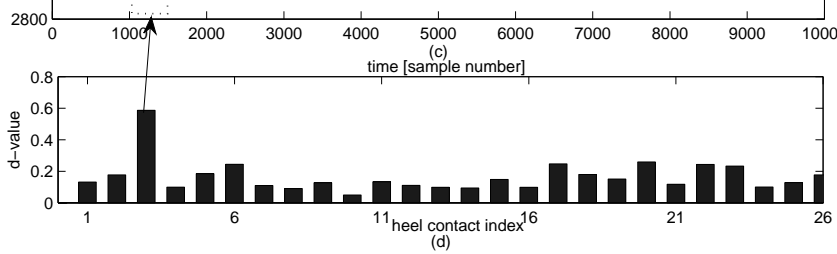

Fig. 10. Example of one patient with only one segment relating to underestimated gait events which is detected accurately. (a) Raw accelerations of the ML, AP and SI axes. (b) Trend removed accelerations of all axes and detected RHCs and LHCs. (c) Interpolated accelerations of the ML axis i.e. same length for the cycles considering right-to-right heel contacts. (d) values of the vector of $\mathbf{d}$ in Algorithm 2 which shows one segment relating to underestimated gait events.

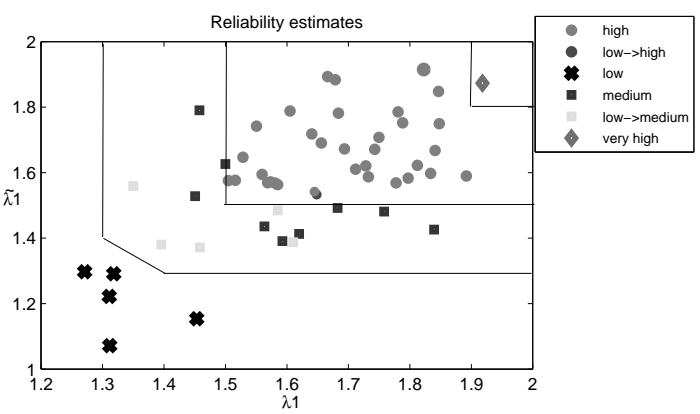

Fig. 11. Rough Partitioning of the reliability estimates for 54 ACL and knee replacement patients based on $\lambda_{1}$ and $\widetilde{\lambda}_{1}$ values (very $\operatorname{high}\left(\widetilde{\widetilde{\lambda}}_{1} \geq\right.$ 1.8 and $\left.\lambda_{1} \geq 1.9\right)$, high $\left(\widetilde{\lambda}_{1} \geq 1.5\right.$ and $\left.\lambda_{1} \geq 1.5\right)$, medium $\left(\widetilde{\lambda}_{1} \geq\right.$ 1.3 and $\lambda_{1} \geq 1.3$ and $\left.\widetilde{\lambda}_{1}+\lambda_{1} \geq 2.7\right)$ ). In the case of detecting overestimation problem and then re-applying the gait analysis platform by setting a threshold on the peaks obtained from dominant oscillation, $v=1$, and $v=1$ were obtained after recall of the algorithm in Algorithm 1. Therefore, for all patients, all final values $v$ and $\widetilde{v}$ are found as $v=1$ and $\widetilde{v}=1.64 .8 \%$ of the estimates have reliability of high or very high.

at different sessions considering gait segments are provided in Table III. Entries with NaN values represent a missing session. In Table III, for each subject, a decrease in gait parameters with an increase in time after surgery is highlighted in blue colour. Considering eight subjects who performed the whole experiment throughout the year and the extracted features, separate clusters of patterns were found for 3 months and 1 year after surgery. However, there were overlaps for the patterns relating to 6 and 9 months post-operatively. By integrating more features from various estimated gait parameters, separation of all stages of the recovery can be investigated in future studies. In general, a decrease in right stride time (increase in speed of walking), step time asymmetry and amplitude asymmetry are important clinical features for observing recovery of the patients. 
TABLE I

A NUMBER OF STUDIES COVERING DIFFERENT WEARABLE SENSING PLATFORMS AND PATIENT POPULATIONS

\begin{tabular}{|c|c|c|c|c|}
\hline Reference & Sensor & $\begin{array}{l}\text { Subjects } \\
\end{array}$ & Study protocol & Clinical Application \\
\hline $\begin{array}{l}\text { Jarchi et al. } \\
{[12,22]} \\
\text { Atallah et al. } \\
{[23,24]} \\
\text { Kwasnicki et al. } \\
{[5]}\end{array}$ & $\begin{array}{l}\frac{\text { single }}{-3 \mathrm{D} \text { accelerometer worn }} \\
\text { on the ear (e-AR) }\end{array}$ & $\begin{array}{l}\text { - healthy subjects[12,22,23,24] } \\
\text { - patients after hip or knee } \\
\text { replacement surgery and patients } \\
\text { with knee Osteoarthritis (OA) [24] } \\
\text { - patients following lower limb } \\
\text { trauma reconstruction [5] }\end{array}$ & $\begin{array}{l}\text { - treadmill walking } \\
{[12,23,24]} \\
\text { - walking in a corridor } \\
{[5,22]}\end{array}$ & $\begin{array}{l}\text { - detection of gait asymmetry } \\
{[22,24]} \\
\text { - psychometric evaluation of a } \\
\text { sensor-based mobility score called } \\
\text { HMS [5] }\end{array}$ \\
\hline $\begin{array}{l}\text { Bamberg et al. } \\
\text { [8] }\end{array}$ & $\begin{array}{l}\frac{\text { multiple }}{\text { - two dual axis }} \\
\text { accelerometer } \\
\text { - three gyroscopes placed } \\
\text { at the back of shoes }\end{array}$ & $\begin{array}{l}\text { - healthy subjects } \\
\text { - PD patients }\end{array}$ & $\begin{array}{l}\text { - walking at self-selected } \\
\text { natural Pace expressed } \\
\text { "free gait" outside } \\
\text { laboratory }\end{array}$ & $\begin{array}{l}\text { validation of GaitShoe system } \\
\text { using healthy and PD patients }\end{array}$ \\
\hline Selles et al. [13] & $\begin{array}{l}\text { multiple } \\
\text { - two uniaxial } \\
\text { accelerometers on the } \\
\text { shank }\end{array}$ & $\begin{array}{l}\text { - healthy subjects } \\
\text { - unilateral transtibial amputees }\end{array}$ & $\begin{array}{l}\text { - walking on straight } \\
\text { track with a Kistler force } \\
\text { platform }\end{array}$ & $\begin{array}{l}\text { validation of gait parameters using } \\
\text { controls and transtibial amputees }\end{array}$ \\
\hline Turcot et al. [14] & $\begin{array}{l}\text { multiple } \\
\text { - two triaxial } \\
\text { accelerometer } \\
\text { - two triaxial gyroscope }\end{array}$ & $\begin{array}{l}\text { - asymptomatic subjects } \\
\text { - patients with knee OA }\end{array}$ & $\begin{array}{l}\text { - walking on straight } \\
\text { track with a Kistler force } \\
\text { platform }\end{array}$ & $\begin{array}{l}\text { discrimination between } \\
\text { asymptomatic subjects and medial } \\
\text { knee OA patients }\end{array}$ \\
\hline $\begin{array}{l}\text { Yoneyama et al. } \\
{[20,21]} \\
\text { Sejdic et al. }[16]\end{array}$ & $\begin{array}{l}\frac{\text { single }}{- \text { trunk mounted }} \\
\text { accelerometer }\end{array}$ & $\begin{array}{l}\text { - healthy subjects } \\
\text { - PD patients }[20,21] \\
\text { - PD and peripheral neuropathy } \\
\text { patients }[16]\end{array}$ & $\begin{array}{l}\text { - walking on level ground } \\
{[20,21]} \\
\text { - treadmill walking }[16]\end{array}$ & $\begin{array}{l}\text { - accelerometry-based evaluation } \\
\text { and assessment of daily gait } \\
\text { characteristics [20,21] } \\
\text { - comparison of accelerometer } \\
\text { features between healthy subjects } \\
\text { and patients groups [16] }\end{array}$ \\
\hline $\begin{array}{l}\text { Huisinga et } \\
\text { al.[25] }\end{array}$ & $\begin{array}{l}\frac{\text { multiple }}{-3 \mathrm{D} \text { accelerometers on }} \\
\text { lower and upper trunk }\end{array}$ & $\begin{array}{l}\text { - healthy subjects } \\
\text { - patients with multiple sclerosis } \\
\text { (MS) }\end{array}$ & $\begin{array}{l}\text { - walking on a foot } \\
\text { hallway }\end{array}$ & $\begin{array}{l}\text { - evaluating gait variability } \\
\text { between MS patients and healthy } \\
\text { controls }\end{array}$ \\
\hline
\end{tabular}

\section{Discussion AND Conclusions}

For developing a gait analysis platform to be used for everyday clinical utilisation, it is essential to give a confidence level to the output estimations. The presented work is the first study to define a direct reliability measure as a feedback for the gait analysis system based on prior knowledge of acceleration characteristics to perform re-analysis of estimated gait events for the data recorded outside the gait laboratory. Such reliability measure can be used not only internally for improving the overall performance and increasing the reliability of subsequently derived gait parameters, but it can also give the user a confidence level for the outputs, suggest a complex gait, repeating the experiment or changing the selected gait segment. In practice, the gait segment often contains noisy acceleration data due to a number of factors. Accurate gait event segmentation is crucial to give clinicians and patients reliable information.

Clinically, some gait parameters can be very important during the recovery of orthopaedic patients. For example, the amount of force exerted on each foot can be used by clinicians and physiotherapists to choose an appropriate treatment and walking aid for the patients. As an example, when analysing the e-AR data of a female patient in this study with left unilateral knee replacement, the gait asymmetry was found to be as a greater towards the left foot. After reviewing her records with the clinician, it was found that the patient had previously right hip replacement. In some cases, the patients were unaware of the imbalance of the forces exerted on their feet. It has been observed that some patients with a large temporal asymmetry in step time were likely to put more weight onto the injured foot.

For some patients, especially the older patients with single-sided total knee replacement, there was a risk that the patient will become a candidate for bilateral knee replacement due to excessive force exerted on the native knee. Therefore, it is important to develop a gait monitoring system with a focus on gait asymmetry parameters, ideally in free-living environment over a long period of time. In this research, subtle changes in e-AR accelerations were detected by employing appropriate signal processing techniques. The main advantage of e-AR sensor is in its bio-inspired designed, consistent sensor placement and patient compliance. For example, it does not matter whether the sensor has been worn on the left ear or right ear and head-movement artifacts can be easily removed by applying signal processing techniques. During the past decade, various sensor platforms such as force-plates, pressure insoles, camera-based system and body worn sensor such as inertial measurement units have been developed for gait analysis, the usage of the such systems inside the clinic have not yet been established. However, the use of a lightweight sensor with consistent sensor placement in this study has shown the potential of the sensor and the analysis platform for gait assessment inside the clinic and a large scale patient data analysis. In addition the memory storage and battery life of the sensor which includes an accelerometer provides a good way of using the sensor for continuous home-based monitoring that is preferable to the use of some wearable sensors such as gyroscopes which result in significant drift over an hour of measurements.

By validating the proposed algorithm in [22] on a large number of datasets of orthopaedic patients' data, it was found that there are more cases with over-estimation problem (comparing to underestimation problem) which can potentially be eliminated by applying the proposed algorithm. In this research, clinically relevant parameters are investigated for 16 patients after lower limb reconstruction. These parameters are directly related to heel contact events. For certain patient groups, toe-off events are also of great importance. Although the reliability evaluation proposed in this paper is based on considering heel contacts only, by having highly accurate estimated heel contacts more accurate estimates will be obtained for toeoff events. By improving the gait analysis platform and producing reliable outputs, it is possible to use all derived gait parameters such as swing, stance and single or double support for potential applications in future studies.

\section{ACKNOWLEDGEMENTS}

This work is supported by the UK's Engineering and Physical Sciences Research Council (ESPRIT Programme Grant, EP/H009744/1). The e-AR sensor is now provided commercially by Sensixa, a spin-off 
TABLE III

CHANGES IN THREE DERIVED GAIT PARAMETERS OF 16 PATIENTS WITH INCREASE IN TIME AFTER SURGERY

\begin{tabular}{|c|c|c|c|c|c|c|c|c|c|c|c|c|}
\hline & \multicolumn{4}{|c|}{ right stride time (seconds) } & \multicolumn{4}{|c|}{ amplitude asymmetry } & \multicolumn{4}{|c|}{ step time asymmetry } \\
\hline & \multicolumn{4}{|c|}{ months after surgery } & \multicolumn{4}{|c|}{ months after surgery } & \multicolumn{4}{|c|}{ months after surgery } \\
\hline & 3 & 6 & 9 & 12 & 3 & 6 & 9 & 12 & 3 & 6 & 9 & 12 \\
\hline 1 & 1.4417 & 1.1025 & 1.1800 & 1.0827 & 0.3151 & 0.3208 & 0.2237 & 0.1376 & 0.5374 & 0.0596 & 0.0401 & 0.0299 \\
\hline 2 & 1.3450 & 1.1217 & 1.0891 & 1.1300 & 0.1422 & 0.0906 & 0.0210 & 0.0637 & 0.1988 & 0.0472 & 0.0357 & 0.0539 \\
\hline 3 & 1.4556 & 1.1900 & 1.2367 & 1.1460 & 0.3923 & 0.1978 & 0.3991 & 0.1693 & 0.1261 & 0.1252 & 0.0655 & 0.0294 \\
\hline 4 & 1.2907 & 1.0713 & 1.0827 & 1.0627 & 0.1132 & 0.0486 & 0.2456 & 0.3218 & 0.3050 & 0.0865 & 0.0271 & 0.0758 \\
\hline 5 & 1.2259 & 1.0344 & 1.0162 & 1.0180 & 0.1714 & 0.8340 & 0.1424 & 0.0897 & 0.0375 & 0.0117 & 0.0071 & 0.0052 \\
\hline 6 & 1.2729 & 1.0420 & 1.0000 & 1.0688 & 0.0611 & 0.0064 & 0.0138 & 0.0185 & 0.1255 & 0.0195 & 0.0051 & 0.0071 \\
\hline 7 & 1.3629 & 1.1230 & 1.1444 & 1.1356 & 0.2003 & 0.1993 & 0.0601 & 0.0332 & 0.2211 & 0.0128 & 0.0277 & 0.0070 \\
\hline 8 & 1.1910 & 1.1440 & 1.1300 & 1.0350 & 0.2787 & 0.1600 & 0.3734 & 0.1715 & 0.0575 & 0.0391 & 0.0115 & 0.0425 \\
\hline 9 & 1.2060 & 1.2478 & 1.2478 & $\mathrm{NaN}$ & 0.1259 & 0.1430 & 0.1430 & $\mathrm{NaN}$ & 0.1814 & 0.2782 & 0.2782 & $\mathrm{NaN}$ \\
\hline 10 & 1.4367 & 1.2150 & 1.2900 & $\mathrm{NaN}$ & 0.1949 & 0.0459 & 0.1794 & $\mathrm{NaN}$ & 0.0236 & 0.0185 & 0.0305 & $\mathrm{NaN}$ \\
\hline 11 & 1.3475 & 1.1400 & 1.0867 & $\mathrm{NaN}$ & 0.4297 & 0.2094 & 0.1639 & $\mathrm{NaN}$ & 0.0313 & 0.1048 & 0.0507 & $\mathrm{NaN}$ \\
\hline 12 & $\mathrm{NaN}$ & 1.2915 & 1.4654 & 1.1563 & $\mathrm{NaN}$ & 0.0039 & 0.0060 & 0.0251 & $\mathrm{NaN}$ & 0.0264 & 0.1426 & 0.0696 \\
\hline 13 & $\mathrm{NaN}$ & 1.5667 & 1.3275 & 1.2338 & $\mathrm{NaN}$ & 0.3890 & 0.0816 & 0.0997 & $\mathrm{NaN}$ & 0.0329 & 0.2792 & 0.2348 \\
\hline 14 & 1.4360 & 1.2583 & $\mathrm{NaN}$ & $\mathrm{NaN}$ & 2.0981 & 0.8789 & $\mathrm{NaN}$ & $\mathrm{NaN}$ & 0.1957 & 0.0671 & $\mathrm{NaN}$ & $\mathrm{NaN}$ \\
\hline 15 & 1.4180 & $\mathrm{NaN}$ & 1.0614 & $\mathrm{NaN}$ & 0.3388 & $\mathrm{NaN}$ & 0.4824 & $\mathrm{NaN}$ & 0.0773 & $\mathrm{NaN}$ & 0.2949 & $\mathrm{NaN}$ \\
\hline 16 & 1.0300 & $\mathrm{NaN}$ & $\mathrm{NaN}$ & $\mathrm{NaN}$ & 0.2591 & $\mathrm{NaN}$ & $\mathrm{NaN}$ & $\mathrm{NaN}$ & 0.1515 & $\mathrm{NaN}$ & $\mathrm{NaN}$ & $\mathrm{NaN}$ \\
\hline
\end{tabular}

TABLE II

ANALYSIS OF ESTIMATIONS FOR RELIABILITY OF LOW AND MEDIUM. $\checkmark$ MEANS THERE IS NOT ANY OVER-ESTIMATION OR UNDER-ESTIMATION PROBLEM. X INDICATES AN UNSOLVED UNDER-ESTIMATION OR OVER-ESTIMATION PROBLEM. $X \longrightarrow \checkmark$ INDICATES ELIMINATION OF UNDER-ESTIMATION OR OVER-ESTIMATION PROBLEM.

\begin{tabular}{|c|c|c|c|}
\hline Subject ID & Reliability & Over-estimation & Under-estimation \\
\hline$\# 15$ & medium & $\checkmark$ & $\checkmark$ \\
\hline \#20 & medium & $\checkmark$ & $\checkmark$ \\
\hline \#38 & medium & $\checkmark$ & $\checkmark$ \\
\hline \#52 & medium & $\checkmark$ & $\checkmark$ \\
\hline$\# 27$ & medium & $\checkmark$ & $\mathbf{X} \rightarrow \checkmark$ \\
\hline$\# 9$ & medium & $\checkmark$ & X \\
\hline \#14 & medium & $\checkmark$ & $\mathbf{X}$ \\
\hline \#49 & medium & $\checkmark$ & $\mathbf{X}$ \\
\hline \#51 & medium & $\checkmark$ & $\mathbf{X}$ \\
\hline \#6 & low $\rightarrow$ medium & $\mathrm{X} \rightarrow \checkmark$ & $\mathbf{X} \rightarrow \checkmark$ \\
\hline \#16 & low $\rightarrow$ medium & $\mathbf{X} \rightarrow \checkmark$ & $\mathbf{X} \rightarrow \checkmark$ \\
\hline \#29 & low $\rightarrow$ medium & $\mathbf{X} \rightarrow \checkmark$ & $\mathbf{X} \rightarrow \checkmark$ \\
\hline \#36 & low $\rightarrow$ medium & $\mathbf{X} \rightarrow \checkmark$ & $\mathbf{X} \rightarrow \checkmark$ \\
\hline \#44 & low $\rightarrow$ medium & $\mathbf{X} \rightarrow \checkmark$ & $X \rightarrow \checkmark$ \\
\hline$\# 19$ & low $\rightarrow$ low & $\bar{X} \rightarrow \checkmark$ & $\bar{X} \rightarrow \checkmark$ \\
\hline \#2 & low $\rightarrow$ low & $\mathbf{X} \rightarrow \checkmark$ & $\mathrm{X}$ \\
\hline \#14 & low $\rightarrow$ low & $\mathbf{X} \rightarrow \checkmark$ & $\mathbf{X}$ \\
\hline \#34 & low $\rightarrow$ low & $\mathbf{X} \rightarrow \checkmark$ & $\mathbf{X}$ \\
\hline \#42 & low $\rightarrow$ low & $\mathbf{X} \rightarrow \checkmark$ & $\mathbf{X}$ \\
\hline
\end{tabular}

company from Imperial College London, for which GZY is a director. The authors would like to thank Louis Atallah, Richard Kwasnicki for providing part of the dataset and also for giving useful comments to improve the quality of the paper.

\section{REFERENCES}

[1] R. M. Kay, S. Dennis, S. Rethlefsen, D. L. Skaggs, and V. T. Tolo, "Impact of postoperative gait analysis on orthopaedic care," Clinical Orthopaedics Related Research, vol. 374, pp. 259-264, 2000.

[2] R. M. Kay, S. Dennis, S. Rethlefsen, R. A. Reynolds, D. L. Skaggs, and V. T. Tolo, "The effect of preoperative gait analysis on orthopaedic decision making," Clinical Orthopaedics Related Research, vol. 372, pp. 217-222, 2000.

[3] B. Lofterd, T. Terjesen, I. Skaaret, A. B. Huse, and R. Jahnsen, "Preoperative gait analysis has a substantial effect on orthopedic decision making in children with cerebral palsy: comparison between clinical evaluation and gait analysis in 60 patients," Acta Orthopaedica, vol. 78, no. 1 , pp. 74-80, 2007.

[4] R. E. Cook, I. Schneider, M. E. Hazlewood, S. J. Hillman, and J. E. Robb, "Gait analysis alters decision-making in cerebral palsy," Journal of Pediatric Orthopedics, vol. 23, no. 3, pp. 292-295, 2003.

[5] R. M. Kwasnicki, S. Hettiaratchy, D. Jarchi, C. Nightingale, M. Wordsworth, J. Simmons, G. Z. Yang, and A. Darzi, "Assessing functional mobility after lower limb reconstruction: A psychometric evaluation of a sensor-based mobility score," Annals of Surgery, doi:10.1097/SLA.0000000000000711, 2014.
[6] E. R. Boyer and B. D. Rooney and T. R. Derrick, "Rearfoot and midfoot or forefoot impacts in habitually shod runners," Med Sci Sports Exerc, vol. 46, no. 7, pp. 1384-1391, 2014.

[7] R. C. Gonzalez, A. M. Lopez, J. Rodriguez-Ura, D. Alvarez, and J. C. Alvarez, "Real-time gait event detection for normal subjects from lower trunk accelerations," Gait Posture., vol. 31, no. 3, pp. 322-325, 2010.

[8] S. J. M. Bamberg, A. Y. Benbasat, D. M. Scarborough, D. E. Krebs, and J. A. Paradiso, "Gait analysis using a shoe-integrated wireless sensor system," IEEE Transactions on information technology in biomedicine, vol. 12, no. 4, pp. 413-423, 2008

[9] J. M. Jasiewicz, J. H. Allum, J. W. Middleton, A. Barriskill, P. Condie, B. Purcell, and R. C. .Li, "Gait event detection using linear accelerometers or angular velocity transducers in able-bodied and spinal-cord injured individuals," Gait Posture, vol. 24, no. 4, pp. 502-509, 2006.

[10] W. Zijlstra and A. L. Hof, "Assessment of spatio-temporal gait parameters from trunk accelerations during human walking," Gait Posture, vol. 18, no. 2, pp. 1-10, 2003

[11] R. R. Torrealba, J. Cappelletto, L. Fermin-Leon, J. C. Grieco, and G. Fernandez-Lopez, "Statistics-based technique for automated detection of gait events from accelerometer signals," Electronics Letters, vol. 46, no. 22 , pp. 1483-1485, 2010.

[12] D. Jarchi, C. Wong, R. M. Kwasnicki, B. Heller, G. Tew, and G.-Z. Yang, "Gait parameter estimation from a miniaturised ear-worn sensor using singular spectrum analysis and longest common subsequence," IEEE Transactions on Biomedical Engineering, vol. 61, no. 4, pp. 1261-1273, 2014.

[13] R. W. Selles, M. A. G. Formanoy, J. B. J. Bussmann, P. J. Janssens, and H. J. Stam, "Automated estimation of initial and terminal contact timing using accelerometers; development and validation in transtibial amputees and controls," IEEE Trans. Neural. Syst. Rehabil. Eng., vol. 13, no. 1, pp. 81-88, 2005.

[14] K. Turcot, R. Aissaoui, K. Boivin, M. Pelletier, N. Hagemeister, and J. A. de Guise, "New accelerometric method to discriminate between asymptomatic subjects and patients with medial knee osteoarthritis during 3-d gait," IEEE Transactions on Biomedical Engineering, vol. 55, no. 4, pp. 1415-1422, 2008.

[15] Y. Wu and S. Krishnan, "Statistical analysis of gait rhythm in patients with parkinson's disease," IEEE Trans Neural Syst Rehabil Eng., vol. 18 , no. 2, pp. 150-158, 2010.

[16] E. Sejdic, K. A. Lowry, J. Bellanca, M. S. Redfern, and J. S. Brach, "A comprehensive assessment of gait accelerometry signals in time, frequency and time-frequency domains," IEEE Transactions on neural systems and rehabilitation engineering, vol. 22, no. 3, pp. 603-612, 2014.

[17] P. Gupta and T. Dallas, "Feature selection and activity recognition system using a single triaxial accelerometer," IEEE Transactions on Biomedical Engineering, vol. 61, no. 6, pp. 1780-1786, 2014.

[18] A. Santa'Anna and N. Wickstrom, "A symbol-based approach to gait analysis from acceleration signals: Identification and detection of gait events and a new measure of gait symmetry," IEEE Transactions on Information Technology in Biomedicine, vol. 14, no. 5, pp. 1180-1187, 2010.

[19] M. Avvenuti, A. Casella, and D. Cesarini, "Using gait symmetry to virtually align a triaxial accelerometer during running and walking," Electronics Letters, vol. 49, no. 2, pp. 120-121, 2013. 
[20] M. Yoneyama, Y. Kurihara, K. Watanabe, and H. Mitoma, "Accelerometry-based gait analysis and its application to parkinsons disease assessment part 1: Detection of stride event," IEEE Transactions on neural systems and rehabilitation engineering, vol. 22, no. 3, pp. 613-622, 2014.

[21] M. Yoneyama, Y. Kurihara, K. Watanabe, and H. Mitoma, "Accelerometry-based gait analysis and its application to parkinsons disease assessment part 2: A new measure for quantifying walking behavior," IEEE Transactions on neural systems and rehabilitation engineering, vol. 21, no. 6, pp. 999-1005, 2013.

[22] D. Jarchi, B. Lo, E. Ieong, D. Nathwani, and G.-Z. Yang, "Validation of the e-AR sensor for gait event detection using the parotec foot insole with application to post-operative recovery monitoring," International workshop on wearable and implantable body sensor networks, BSN 2014.

[23] L. Atallah, A. Wiik, G. G. Jones, B. Lo, J. P. Cobb, A. Amis, and G. Z. Yang, "Validation of an ear-worn sensor for gait monitoring using a force-plate instrumented treadmill," Gait Posture, vol. 35, no. 4, pp. 674-676, 2012.

[24] L. Atallah, A. Wiik, B. Lo, J. P. Cobb, A. A. Amis, and G.-Z. Yang, "Gait asymmetry detection in older adults using a light ear-worn sensor," Physiol. Meas., vol. 35, no. 5, pp. N29-N40, 2014.

[25] J. M. Huisinga, M. Mancini, R. J. St. George, and F. B. Horak, "Accelerometry reveals differences in gait variability between patients with multiple sclerosis and healthy controls," Annals of Biomedical Engineering, vol. 41, no. 8, pp. 1670-1679, 2013.

[26] B. Lo, S. Thiemjarus, A. Panousopoulou, and G. Z. Yang, "Bioinspired design for body sensor networks," IEEE Signal Processing Magazine Life Science Column, 2012.

[27] G. Z. Yang, Body Sensor Networks., Berlin, Germany: Springer-Verlag, 2014.

[28] Parotec System instruction manual, Paromed Medizintechnik GmbH., Neubeuern, Germany, 1997.

[29] D. Jarchi, L. Atallah, and G. Z. Yang, "Transition detection and activity classification from wearable sensors using singular spectrum analysis," Ninth international workshop on wearable and implantable body sensor networks, BSN 2012, pp. 136-141, 2012.

[30] N. Golyandina, V. Nekrutkin, and A. Zhigljavsky, "Analysis of timeseries structure: SSA and related techniques," Chapman and Hall/CRC, 2001.

[31] S. Sanei, Adaptive Processing of Brain Signals, John Wiley and Sons, 2013.

[32] V. G. Moskvina and A. Zhigljavsky, "Application of singular-spectrum analysis to change-point detection in time series," School of Mathematics, Cardiff University, 2003a.

[33] V. G. Moskvina and A. Zhigljavsky, "An algorithm based on singular spectrum analysis for change-point detection," Communications in Statistics: Simulation and Computation, vol. 32, no. 2, pp. 319-352, 2003. 